\title{
Microfluidics-based optimization of neuroleukin-mediated regulation of articular chondrocyte proliferation
}

\author{
KANG TIAN, WEILIANG ZHONG, YINGQIU ZHANG, BAOSHENG YIN, WEIGUO ZHANG and HAN LIU \\ Department of Orthopaedics, First Affiliated Hospital, Institute of Cancer Stem Cell, \\ Dalian Medical University, Dalian, Liaoning 116044, P.R. China
}

Received January 22, 2015; Accepted September 14, 2015

DOI: $10.3892 / \mathrm{mmr} .2015 .4540$

\begin{abstract}
Due to the low proliferative and migratory capacities of chondrocytes, cartilage repair remains a challenging clinical problem. Current therapeutic strategies for cartilage repair result in unsatisfactory outcomes. Autologous chondrocyte implantation (ACI) is a cell based therapy that relies on the in vitro expansion of healthy chondrocytes from the patient, during which proliferation-promoting factors are frequently used. Neuroleukin (NLK) is a multifunctional protein that possesses growth factor functions, and its expression has been associated with cartilage development and bone regeneration, however its direct role in chondrocyte proliferation remains to be fully elucidated. In the current study, the role of NLK in chondrocyte proliferation in vitro in addition to its potential to act as an exogenous factor during ACI was investigated. Furthermore, the concentration of NLK for in vitro chondrocyte culture was optimized using a microfluidic device. An NLK concentration of $12.85 \mathrm{ng} / \mathrm{ml}$ was observed to provide optimal conditions for the promotion of chondrocyte proliferation. Additionally, NLK stimulation resulted in an increase in type II collagen synthesis by chondrocytes, which is a cartilaginous secretion marker and associated with the phenotype of chondrocytes. Together these data suggest that NLK is able to promote cell proliferation and type II collagen synthesis during in vitro chondrocyte propagation, and thus may serve as an exogenous factor for ACI.
\end{abstract}

\section{Introduction}

Due to the lack of vascularization in articular cartilage and the low proliferative and migratory capacities of chondrocytes, cartilage remains a challenging tissue to repair (1-4).

Correspondence to: Professor Weiguo Zhang or Professor Han Liu, Department of Orthopaedics, First Affiliated Hospital, Institute of Cancer Stem Cell, Dalian Medical University, 9 West Section Lvshun South Road, Dalian, Liaoning 116044, P.R. China

E-mail: dlmedu@outlook.com

E-mail: liuhan@dlmedu.edu.cn

Key words: neuroleukin, autocrine motility factor, microfluidics, articular chondrocyte
Current clinical treatment strategies involve mosaicplasty, micro-fracture, periosteum or perichondrium transplantation and fresh osteochondral allograft implantation (5). These strategies appear promising, however the long term outcomes are unsatisfactory $(3,6,7)$. Tissue engineering has provided alternative possibilities for hyaline cartilage repair using cell based therapy, utilizing chondrocytes or adult stem cells combined with synthetic substrates and bioactive factors to prepare for the functional replacement of hyaline cartilage (8-10). Bioactive factors have been widely utilized in cell based therapy to promote cell proliferation and the production of the extracellular matrix, however current therapeutic options are far from optimal and the anticipated outcomes are rarely achieved (11). Overall, it remains to be fully understood how improvements in chondrocyte amplification may be achieved with the maintenance of the chondrocytic phenotype. The aim is to generate active and phenotypically stabilized tissue engineered cartilage in order to treat cartilage lesions.

Neuroleukin (NLK), a neurotrophic factor of spinal and sensory neurons, is additionally a growth factor in mouse salivary glands, and promotes the survival of peripheral and central neurons in culture. NLK is additionally known as autocrine motility factor (AMF) and phosphoglucose isomerase (PGI) $(12,13)$, where the peptide sequences of NLK, AMF and PGI indicate that they are the same protein, with the differing names assigned based on previous functional analyses. NLK is secreted by tumor cells and functions as a cytokine to promote migration, invasion and proliferation of tumor cells (14-16). This paracrine function of NLK is further supported by studies on the migration of vascular endothelial cells which propagate and form new capillaries during tumor angiogenesis, in addition to the proliferation and migration of fibroblasts treated with NLK $(17,18)$. Of note, Zhi et al (19) reported differential expression of NLK in osseous tissue and during differentiation, with elevated levels in superficial articular chondrocytes, proliferating chondrocytes, fibroblasts and osteoblasts within the fracture callus, however, NLF was absent in terminally differentiated hypertrophic chondrocytes or osteocytes. These studies suggest that NLK is involved in cartilage development and bone regeneration. In the current study, the effect of NLK on the proliferation of isolated rat articular chondrocytes was investigated in vitro and the working concentrations of NLK were optimized using a microfluidic device. 


\section{Materials and methods}

Antibodies and reagents. The following antibodies were used in immunofluorescent analyses: Mouse anti-collagen type II polyclonal antibody (1:4,000; cat. no. BA0533; Wuhan Boster Biological Technology, Ltd., Wuhan, China), Alexa Fluor ${ }^{\circledR} 488$ goat anti-mouse secondary antibody (1:1,000; cat. no. A11001; Invitrogen Life Technologies, Carlsbad, USA). Human recombinant NLK/PGI full length protein was obtained from Abcam (Cambridge, UK; cat. no. ab87625). Trypsin was purchased from Gibco Life Technologies (Carlsbad, USA), collagenase type II from Sigma-Aldrich (St. Louis, MO, USA), and the Cell Cycle Detection kit was from Nanjing KeyGen Biotech Co., Ltd. (Nanjing, China; cat. no. KGA511).

Chondrocyte isolation and cell culture. All experimental procedures were approved by the Institutional Committee of Animal Use and Protection (Dalian Medical University, Dalian, China). A total of 12 Sprague-Dawley rats were used in the present study, which were provided by the Experimental Animal Center of the Dalian Medical University. The rats were anesthetized with $3.6 \%$ chloral hydrate $(1 \mathrm{ml} / 100 \mathrm{~g}$; Kermel, Tianjin, China) by intraperitoneal injection, prior to sacrifice by cervical vertebra dislocation. The surrounding muscles of the knee joints were removed to expose the articular cartilage, which was collected using curved tissue scissors. Cartilage was separated from the femoral heads and femoral condyles of 1 month-old male Sprague-Dawley rats and cut into $1.5 \mathrm{~mm}^{3}$ sections using a surgical blade in sterile phosphate-buffered saline (PBS; Beijing Solarbio Science \& Technology Co., Ltd., Beijing, China). Primary chondrocytes were isolated by digestion with $0.2 \%$ type II collagenase for $4 \mathrm{~h}$ at $37^{\circ} \mathrm{C}$ in an agitating water bath and resuspended in Dulbecco's modified Eagle's medium/F-12 (GE Healthcare Life Sciences, Logan, UT, USA) containing $10 \%$ fetal bovine serum (FBS; Gibco Life Technologies). Cells were cultured in a humidified atmosphere at $37^{\circ} \mathrm{C}$ with $5 \% \mathrm{CO}_{2}$. Chondrocytes were trypsinized by $0.25 \%$ trypsin for subcultures.

Microfluidic device design and fabrication. The microfluidic device in the current study provided a pyramid shaped concentration gradient generator (CGG) and a downstream cell culture module. The design and operation of the microfluidic device are presented in Fig. 1. The device had two inlets, one outlet and three cell culture chambers connected to resistance channels. Each chamber was $100 \mu \mathrm{m}$ in height, $400 \mu \mathrm{m}$ in width and $1,000 \mu \mathrm{m}$ in length. A previous study observed that the liquid exhibited laminar flow when it passed through the serpentine channel of the microfluidic chip according to the Reynolds effect when the capillary diameter was approximately $100 \mathrm{~nm}$ (20). All devices were fabricated using conventional micro-fabrication techniques involving SU-8 photolithography and polydimethylsiloxane (PDMS) soft lithography. The transparency mask was designed by AutoCAD 2014 (Autodesk, Inc., San Rafael, CA, USA) and was used in 1:1 contact photolithography with an SU-8 photoresist consisting of patterned photoresist on a silicon wafer. Positive replicas with embossed channels were fabricated by molding PDMS against the master. The PDMS replica and a clean glass substrate were irreversibly sealed using oxygen plasma (2 Torr, $100 \mathrm{~W}$ ) for $1 \mathrm{~min}$.
Microfluidic chip operation. The inlets of the device were connected to a syringe pump (model LSP02-1B; Baoding Longer Precision Pump Co., Ltd., Baoding, China) to drive fluid flow. The outlet of the device was connected to a culture medium reservoir containing DMEM/F12. Culture media with and without NLK were simultaneously infused into the microfluidic device from the two inlets, with $2 \%$ serum containing culture medium into the left and $2 \%$ serum medium with $30 \mathrm{ng} / \mathrm{ml} \mathrm{NLK}$ into the right. The concentration gradient of NLK was established within $30 \mathrm{sec}$. The flow speed was controlled at $0.1 \mathrm{ml} / \mathrm{min}$. The device was stored in an incubator at $37^{\circ} \mathrm{C}$ with $5 \% \mathrm{CO}_{2}$ during the culture period.

$C G G$ performance validation. By using this chip, solutions from inlets were repeatedly split at the nodes, combined with neighboring streams in a laminar fashion, and mixed by diffusion in serpentine channels. As a result, the solutions were continuously diluted and a series of concentrations was produced in the outlets of the CGG. In the current study, solution A (concentration 0 ) and solution B (concentration X) were injected into the CGG via the 2 inlets simultaneously, and the concentrations in the 8 outlets were as follows: $0,1 / 7 \mathrm{X}$, 2/7X, 3/7X, 4/7X, 5/7X, 6/7X and X. Fluorescein isothiocyanate dextran (FITC-dextran) with a molecular weight of 20,000 Da (Sigma-Aldrich) was used as a probe for the CGG performance validation according to a previous study (18). The fluorescence intensity of FITC-dextran at the 8 outlets of the CGG were imaged by confocal laser scanning microscopy (model TCSSP5; Leica Microsystems GmbH, Wetzlar, Germany) and quantified using Image-Pro Plus 6.0 software (Media Cybernetics, Inc., Rockville, MD, USA). The intensities were compared with the theoretical values achieved by the equation, and correlation factors were calculated. All the experiments were repeated three times.

Cell cycle analysis. Cell cycle phase distribution was determined by flow cytometry using propidium iodide (PI; Nanjing KeyGen Biotech Co., Ltd.) staining. For cell synchronization, cells were starved for $12 \mathrm{~h}$ prior to NLK treatment. Following $24 \mathrm{~h}$ culture in $2 \%$ FBS medium and 24/48 h NLK treatment, cells were harvested, fixed in $70 \%$ ice-cold ethanol for $12 \mathrm{~h}$ at $4^{\circ} \mathrm{C}$, stained in $50 \mu \mathrm{g} / \mathrm{ml}$ PI supplemented with $1 \mathrm{mg} / \mathrm{ml}$ RNase (Nanjing KeyGen Biotech Co., Ltd.) and 0.1\% Triton X-100 (Sigma-Aldrich), and analyzed by flow cytometry (Accuri C6; BD Biosciences, Franklin Lakes, NJ, USA). The histogram was used to present the percentage of cells in the $\mathrm{G}_{1}$, $\mathrm{S}$ and $\mathrm{G}_{2} / \mathrm{M}$ phases. Cell cycle distribution was analyzed by FlowJo 10.0.6 software (FlowJo, LLC., Ashland, OR, USA).

Immunofluorescence. Cells were seeded $\left(0.8 \times 10^{5}\right.$ cells/well $)$ on sterile glass coverslips $(0.8 \times 0.8 \mathrm{~cm})$ and cultured in 6 -well plates until $70 \%$ confluent. Cells were washed twice with PBS, fixed with $4 \%$ formaldehyde (Beijing Solarbio Science \& Technology Co., Ltd.) for $15 \mathrm{~min}$ and permeabilized with $0.1 \%$ Triton X-100 for 15 min, prior to blocking with 5\% bovine serum albumin (Beijing Solarbio Science \& Technology Co., Ltd.) in PBS for $1 \mathrm{~h}$ at room temperature. Cells were incubated with primary antibodies overnight at $4^{\circ} \mathrm{C}$. Subsequently, cells were incubated with fluorescent secondary antibodies at room temperature for $1 \mathrm{~h}$ prior to 
A

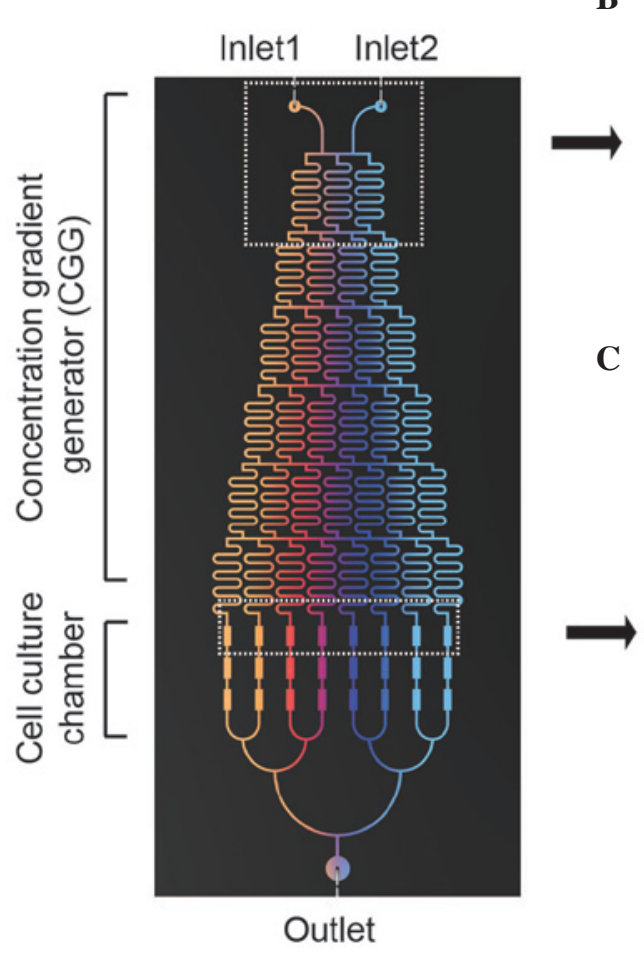

B

C
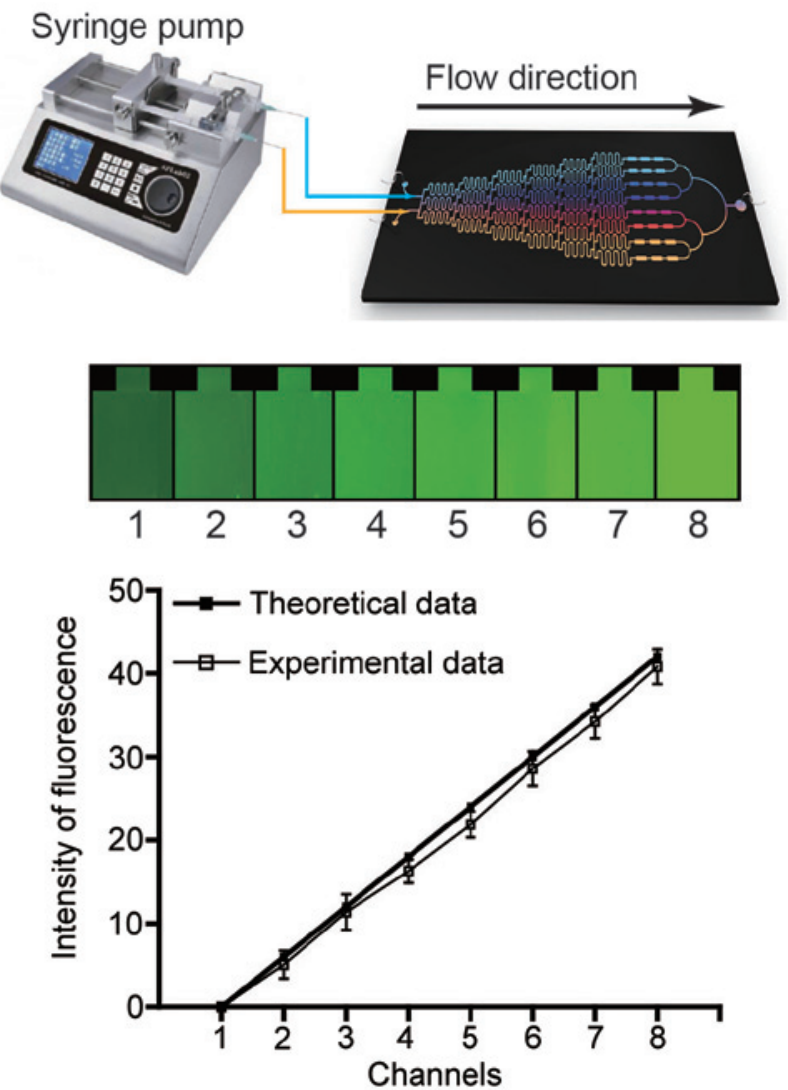

Figure 1. Microfluidic device design and CGG performance validation. (A) The microfluidic device is composed of an upstream CGG and downstream parallel cell culture chambers. From two inlets, represented by different colors, solutions are injected into the device and are gradually diluted to form a gradient. (B) A syringe pump is connected to the microfluidic device and culture medium with/without neuroleukin is pumped into the inputs of the microfluidic device. (C) Images of FITC-dextran in the downstream chambers of the eight outlets from the confocal laser scanning microscope. The experimental intensities of FITC-dextran were quantified and compared with theoretical data. Values are presented as the mean \pm standard deviation. CGG, concentration gradient generator; FITC, fluorescein isothiocyanate.

staining with 4',6-diamidino-2-phenylindole and imaging using a fluorescence microscope (model BX53; Olympus Corporation, Tokyo, Japan).

Observation of chondrocyte morphology. After six days of culture in a microfluidic device (designed and fabricated by Dalian University of Technology, Dalian, China) with various concentrations of NLK $(0,4.28,8.57,12.85,17.15$, $21.42,25.71$ and $30 \mathrm{ng} / \mathrm{ml}$ ), total chondrocyte numbers were counted from several fields and morphological changes of the chondrocytes were observed using a phase contrast microscope (Olympus cx31; Olympus Corporation). Micrographs were captured from the cell culture chambers of the eight groups.

\section{Results}

Microfluidic device design and gradient generation. Dilution networks and micro-scale cell culture chambers were incorporated into a single microfluidic device, which is capable of producing multiple gradient concentrations for on-chip cell culture assays. A series of solutions with different concentrations was formed at the outlets of the CGG $(0,4.28$, 8.57, 12.85, 17.15, 21.42, 25.71 and $30 \mathrm{ng} / \mathrm{ml}$; Fig. 1A). The fluorescent probe FITC-dextran was used as an indicator for estimating the gradient produced by the CGG. From the two inlets, FITC-dextran was simultaneously infused into the CGG by a syringe pump and the resulting gradients (Fig. 1C) were imaged and quantified. A total of eight FITC-dextran gradient profiles were produced from gradient generators spanning the two inlet concentrations (Fig. 1B). The fluorescence intensities of FITC-dextran in the junctions between the CGG and the cell culture chambers were quantified, corrected by subtracting the background fluorescence and compared with the theoretically obtained gradient. A good association (correlation coefficient $=0.9953$ ) was observed between the experimental and theoretical data, as presented in Fig. 1C.

Microfluidic analyses of chondrocyte proliferation with NLK. To evaluate the effect of NLK on chondrocyte proliferation, isolated articular chondrocytes from Sprague-Dawley rats were applied to the above-mentioned microfluidic chip with an NLK concentration gradient. Following 6 days of culture, the morphology of chondrocytes was imaged using phase contrast microscopy (Fig. 2), and a dose-dependent proliferative profile was observed following treatment with NLK between 0 and $12.85 \mathrm{ng} / \mathrm{ml}$ (Fig. 3A). Culture medium with $2 \%$ serum was used as the control. Among the eight concentrations $(0,4.28,8.57,12.85,17.15,21.42,25.71$ and $30 \mathrm{ng} / \mathrm{ml})$, the proliferation rate plateaued at $12.85 \mathrm{ng} / \mathrm{ml}$. The $12.85 \mathrm{ng} / \mathrm{ml}$ NLK group exhibited a 3.96-fold increase in chondrocyte 


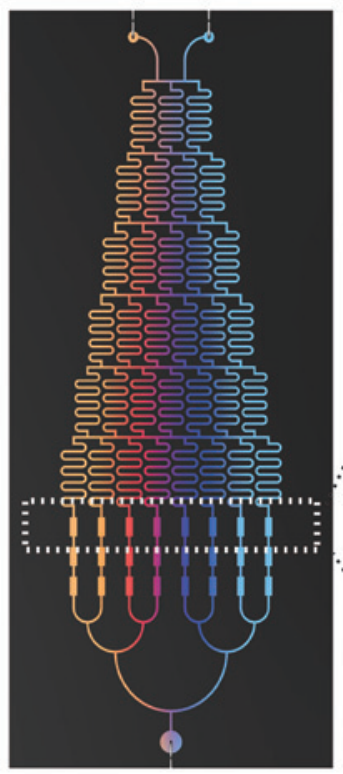

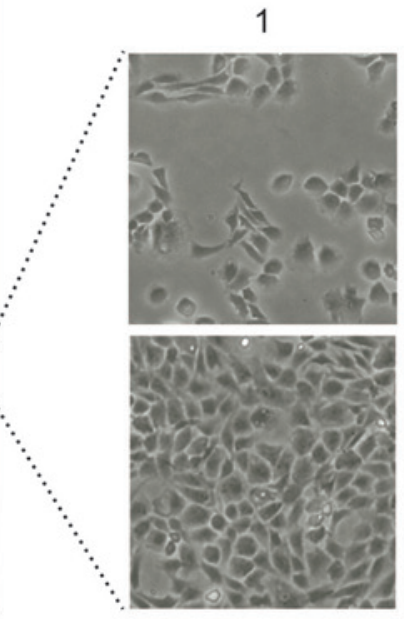

5
2
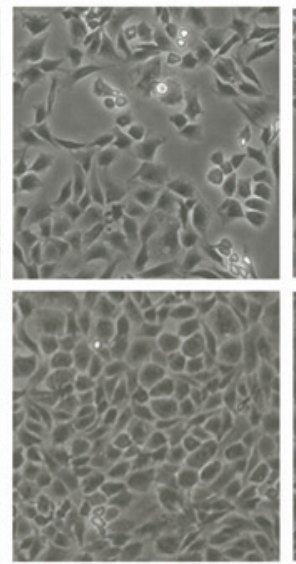

6
3
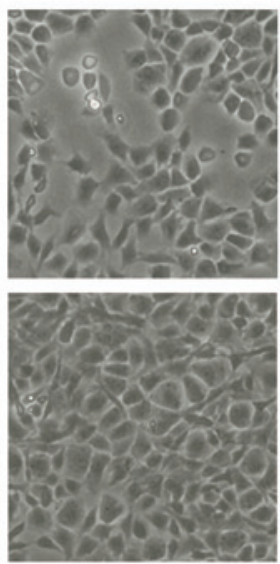

7
4
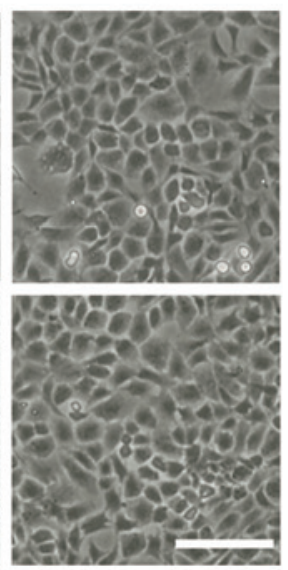

8

Figure 2. Morphology of chondrocytes following 6 days of culture with different concentrations of neuroleukin. The number of chondrocytes was counted on the chips directly with phase contrast microscopy following 6 days of culture. The micrographs $1-8$ were taken from the cell culture chamber of the eight groups. Scale bar $=100 \mu \mathrm{m}$.

A

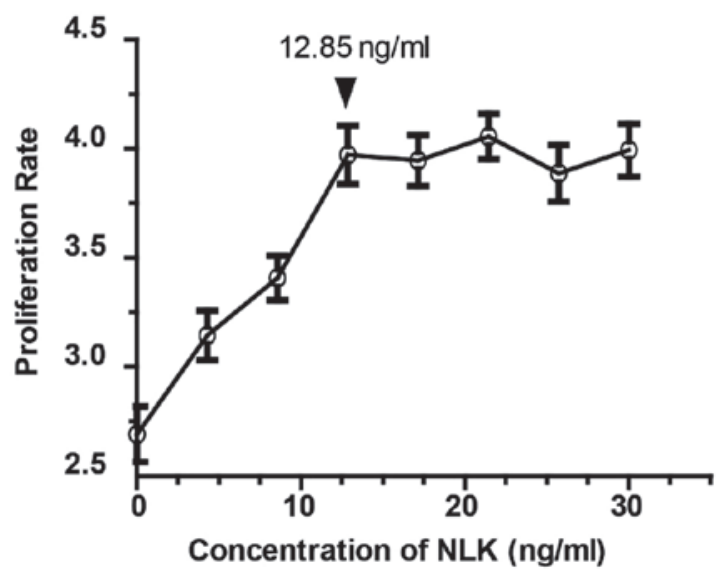

B

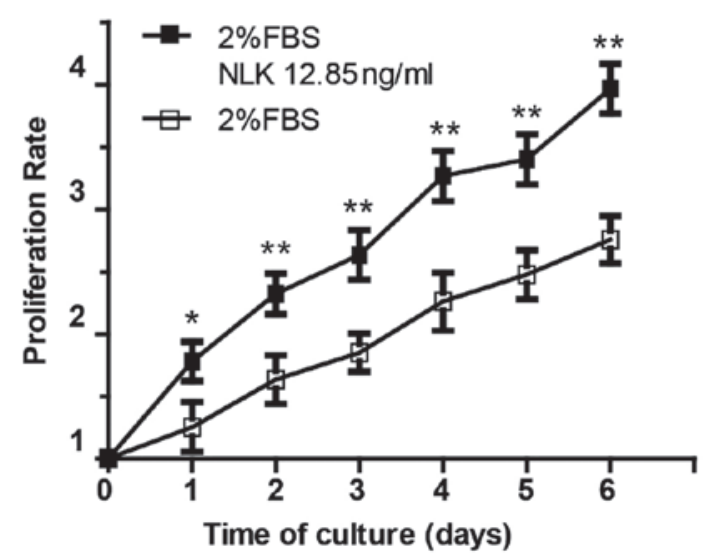

Figure 3. Chondrocyte proliferation following treatment with NLK. (A) NLK stimulates chondrocyte proliferation in a dose-dependent manner up to $12.85 \mathrm{ng} / \mathrm{ml}$ (3.96-fold), with no further promotion observed at higher concentrations (up to $30 \mathrm{ng} / \mathrm{ml}$ ). (B) The growth profile of chondrocytes following $12.85 \mathrm{ng} / \mathrm{ml} \mathrm{NLK}$ treatment during 6 days of culture. Values are presented as the mean \pm standard deviation. ${ }^{*} \mathrm{P}<0.05$ and ${ }^{* * *} \mathrm{P}<0.01$. NLK, neuroleukin; FBS, fetal bovine serum.

proliferation (Fig. 3A), with a statistically significant difference compared with the control group $(\mathrm{P}<0.01)$. However, there was no statistically significant difference between the $12.85 \mathrm{ng} / \mathrm{ml} \mathrm{NLK}$ group and the higher concentrations ( $P>0.05)$. The growth curve of the chondrocytes following $12.85 \mathrm{ng} / \mathrm{ml}$ NLK stimulation is presented in Fig. 3B.

NLK increases the proportion of proliferative state chondrocytes. To further characterize the pro-proliferative effect of NLK on chondrocytes, flow cytometry was conducted to assess cell cycle distribution. Chondrocytes were starved with serum-free medium for $12 \mathrm{~h}$ to synchronize the cells, followed by treatment with $2 \%$ FBS for $24 \mathrm{~h}$ and $12.85 \mathrm{ng} / \mathrm{ml} \mathrm{NLK}$ with $2 \%$ FBS for 24 and $48 \mathrm{~h}$. Cells were collected and analyzed post treatment. As presented in Fig. 4A, an accumulation of
NLK-treated cells in the $S$ phase of the cell cycle was observed compared with the control cells at 24 and $48 \mathrm{~h}$. In addition, the $\mathrm{G}_{2} / \mathrm{M}$ phase distribution was increased following NLK treatment. Quantification indicated that the percentage of sub $\mathrm{G}_{1}$ phase cells was reduced from 82 to $61 \%$, and the proportion of cells in $S$ and $G_{2} / M$ phases was significantly greater in NLK-treated cells $(\mathrm{P}<0.01$; Fig. 4B). These data further indicate that NLK promotes proliferation of articular chondrocytes in vitro.

NLK stimulates type II collagen synthesis in chondrocytes. As demonstrated above, NLK is able to serve as a bioactive molecule for chondrocytes. Therefore, it was investigated whether NLK is involved in phenotype maintenance of chondrocytes. To determine the response of chondrocytes to 
A

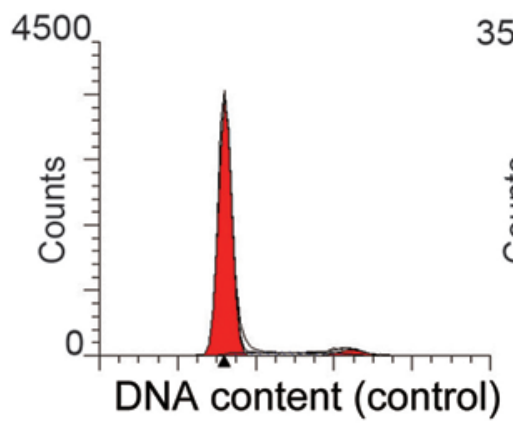

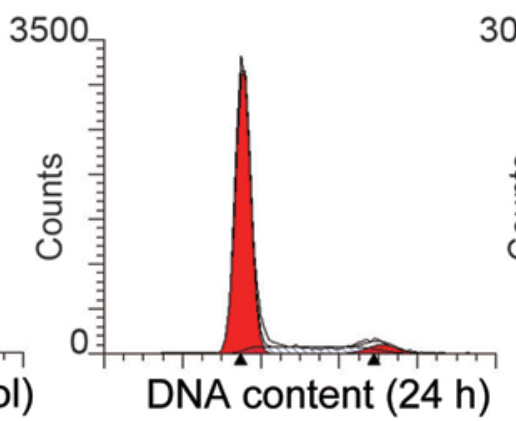

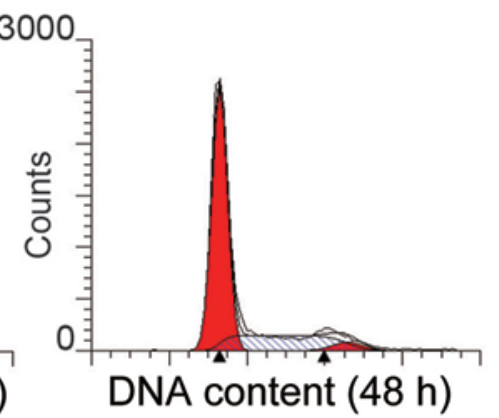

B

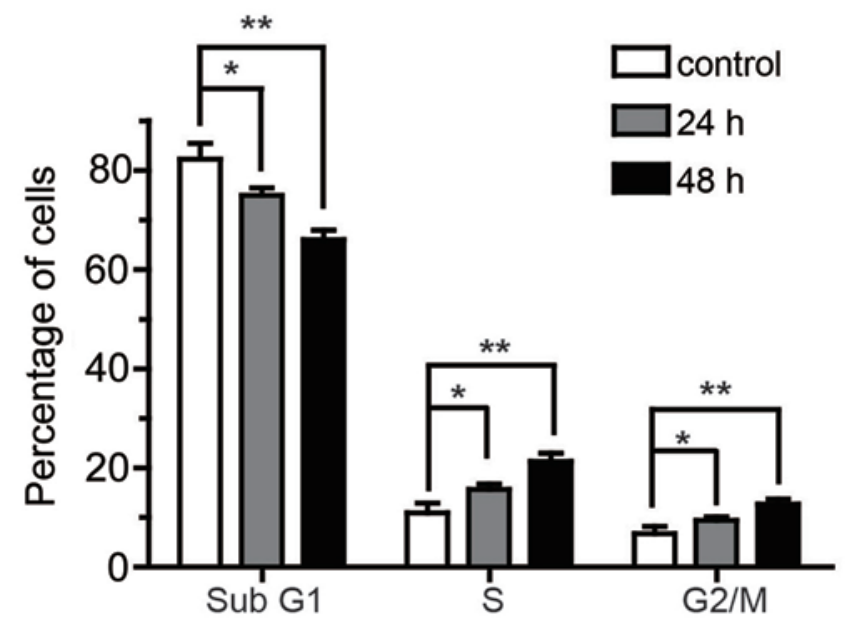

Figure 4. NLK increases the proportion of proliferative chondrocytes. Chondrocytes were treated with NLK for $24 / 48 \mathrm{~h}, 24 \mathrm{~h} 2 \%$ fetal bovine serum treatment was used as the control. Cells were stained with propidium iodide, prior to flow cytometric analysis. (A) Representative histograms from three independent

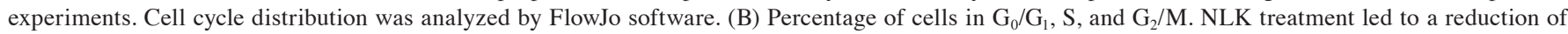
cells in the $G_{0} / G_{1}$ phase, however an increase of cells in the $S$ and $G_{2} / M$ phases. Quantification from 3 independent experiments. Values presented are the mean \pm standard deviation. ${ }^{*} \mathrm{P}<0.05$ and ${ }^{* *} \mathrm{P}<0.01$ vs. control. NLK, neuroleukin.

A
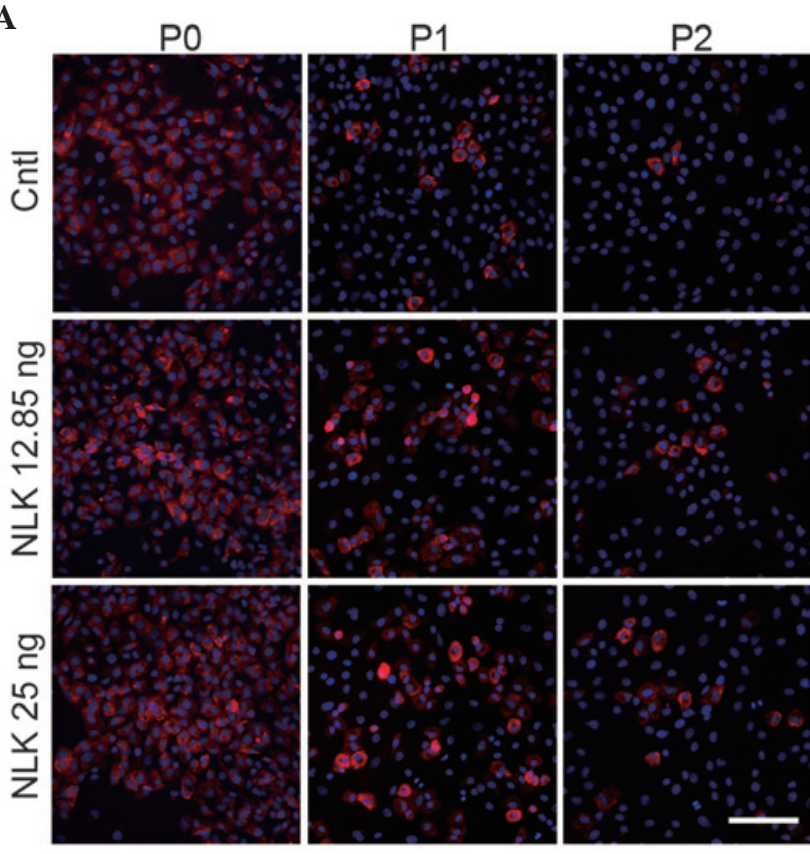

B

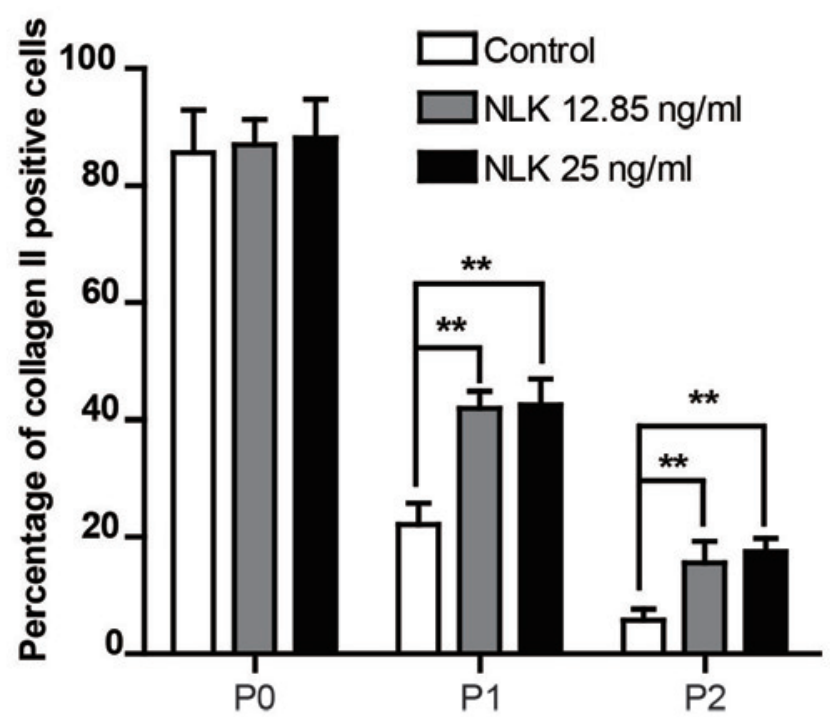

Figure 5. NLK stimulates type II collagen synthesis in chondrocytes. (A) P0, P1 and P2 chondrocytes were cultured with or without NLK (12.85 and 25 ng/ml) and type II collagen expression was detected with immunofluorescence. (B) The percentage of type II collagen positive chondrocytes was calculated. NLK stimulates collagen II synthesis in P1 and P2 cells compared with the control. Quantification from 3 independent experiments. Values presented are the mean \pm standard deviation. ${ }^{* *} \mathrm{P}<0.01$ vs. control. Scale bar $=100 \mu \mathrm{m}$. NLK, neuroleukin; $\mathrm{P}$, passage. 
NLK on type II collagen synthesis during in vitro culture, immunofluorescence analysis was conducted. Chondrocytes were examined from passage 0 ( $\mathrm{P} 0 ; 24 \mathrm{~h}$ following isolation) to passage 2 (P2; 6-7 days following isolation) by staining with the type II collagen antibodies. As presented in Fig. 5A, following $12 \mathrm{~h}$ serum-starvation, $\mathrm{P} 0, \mathrm{P} 1$ and $\mathrm{P} 2$ chondrocytes were cultured with or without NLK (12.85 and $25 \mathrm{ng} / \mathrm{ml})$, and cells with positive type II collagen stains were counted. A reduction in the fluorescent signal of collagen II was observed during prolonged culture in vitro due to the dedifferentiation of primary chondrocytes, characterized by a change from the expression and secretion of Collagen I instead of Collagen II. A significant increase in collagen II positive cells was observed following NLK treatment in P1 (22-41\%) and P2 (5-17\%) groups compared with the control (without NLK). However, no significant difference was observed in P0 or between NLK treatment groups (Fig. 5B). A reduction in the fluorescent signal was observed during prolonged culture in vitro due to dedifferentiation of primary chondrocytes.

\section{Discussion}

Clinical management regarding cartilage repair may be classified into three main categories: Marrow stimulation-based, osteochondral transplantation, and cell-based repair techniques (5). ACI is a cell-based tissue regeneration technique, representing a novel biological approach for the treatment of cartilage defects (21-23). However, due to dedifferentiation of autologous chondrocytes during expansion in vitro and following implantation, ACI may subsequently result in scar tissue akin to fibrocartilage $(22,24)$. Therefore, studies have attempted to combine ACI with biomaterials of natural or synthetic origin as scaffolds, together with the use of bioactive factors providing proliferative stimuli, in order to reconstruct a microenvironment in vitro which allows the cells to grow as in their native tissue $(25,26)$. Bioactive factors function as key components in cell-based therapy. Growth factors that exist in or are secreted by bone marrow mesenchymal stem cells (BMSCs) and chondrocytes such as bone morphogenic protein, vascular endothelial growth factor and transforming growth factor- $\beta$ do not only upregulate chondrogenic markers, however, additionally are associated with ossification (27-29). This may lead to the formation of fibrocartilage with inferior mechanical properties and limited durability $(29,30)$.

NLK is a multifunctional protein with intra- and extracellular functions $(13,31,32)$. It has been studied in neoplastic cells and their normal counterpart cells for the expression, secretion and distribution, suggesting that a wide range of normal and neoplastic cells express and secrete NLK (33-35). Despite the extensive investigation of the pro-metastatic effects in tumor cells, the role of NLK in various normal tissues remains to be fully elucidated. The expression of NLK has been reported to be associated with cerebellar and hippocampal neurons, which are implicated in memory and learning $(36,37)$. NLK is reported to stimulate migration, propagation and new capillary formation for human umbilical vein endothelial cells $(37,38)$. In addition, fibroblasts gain increased proliferative and migratory abilities following treatment with NLK (17). These results provide evidence that NLK may act as a secreted factor to promote cell motility and proliferation in normal tissues. In a previous study, differential mRNA display was used and RNA populations isolated from osseous tissues were compared, from which it was reported that NLK was involved in cartilage development and bone regeneration (21). A high level of NLK expression was identified in superficial articular chondrocytes in 1, 4 and 8-month-old normal mice, in addition to in proliferating chondrocytes. However, in hypertrophic chondrocytes NLK is not detected (19). Furthermore, NLK expression was augmented during fracture healing. From day 3 post fracture, strong NLK labeling was detected in osteoblasts of the newly formed trabecular bone, proliferating chondrocytes of the soft callus and fibrous periosteum. In the late stage of fracture healing, immature osteocytes and proliferating chondrocytes had differentiated towards mature osteocytes and hypertrophic chondrocytes, when the expression of NLK was reduced (19). The spatial and temporal expression of NLK in cartilage and osteoblast development indicates that NLK serves a role during cartilage development.

Microfluidics, known as "lab-on-a-chip", brings the benefits of integration, miniaturization and automation to numerous research areas $(39,40)$. As a novel cell culture platform, microfluidics provides spatial and temporal control of exogenous stimuli via the integration of various functional units (41). Furthermore, microfluidic devices have been successfully used for the long-term culture of cell lines and primary cells $(42,43)$. By using microfluidic chips, experimental conditions are flexible and may be optimized by changing either the type of growth factor or the concentration of the input. Additionally, microfluidics make it possible to perform parallel experiments to optimize conditions whilst using a limited number of cells. A previous study reported data generated from a microfluidic platform, which included the effects of fluid mechanics on articular chondrocytes and BMSCs and the optimization of biofactor concentrations $(20,44)$. As NLK is additionally implicated in tumor cell physiology, its overdosage during in vitro chondrocyte expansion may pose a risk of adverse side effects. In order to maximize its positive effects on chondrocyte proliferation while avoiding detrimental side effects, the current study sought to use microfluidics to optimize the concentration of NLK in chondrocyte in vitro propagation. Using a microfluidic device, 8 concentrations of NLK were generated precisely in $30 \mathrm{sec}$, and the effect of a range of concentrations of NLK on chondrocyte proliferation was evaluated over a 6 day period. A minimal concentration of $12.85 \mathrm{ng} / \mathrm{ml}$ NLK was demonstrated to affect chondrocyte growth, leading to a $\sim 4$-fold increase. This effect of NLK was further supported by the flow cytometry results of cell cycle analysis (Fig. 4).

Dedifferentiation commonly occurs during in vitro expansion of chondrocytes, during which they gradually lose their typical round shape and acquire a spindly fibroblast-like form, with concurrent reductions in cartilaginous protein synthesis such as type II collagen $(29,45,46)$. In the current study, immunofluorescence was conducted to examine the effect of NLK on the synthesis of type II collagen by chondrocytes. Treatment with NLK leads to a significant increase in the number of positively stained cells relative to untreated cells following in vitro passaging, indicating an inhibitory effect of NLK on dedifferentiation. The optimized concentration of $12.85 \mathrm{ng} / \mathrm{ml}$ was not significantly different compared with a 
saturated concentration of $25 \mathrm{ng} / \mathrm{ml}$, confirming the data from the microfluidic device.

In the present study, NLK was reported to act as an exogenous factor to promote the proliferation of articular chondrocytes during in vitro culture. A microfluidic device was used to evaluate a range of concentrations of NLK on chondrocyte proliferation, with a concentration of $12.85 \mathrm{ng} / \mathrm{ml}$ demonstrated to be optimal. Furthermore, this concentration of NLK may additionally prevent dedifferentiation of cultured chondrocytes in vitro. Future studies will aim to provide mechanistic insights into NLK regulation of chondrocyte growth, in addition to assessing the application of NLK during ACI.

\section{Acknowledgements}

The current study was supported by the National Natural Science Foundation of China (grant nos. 81371706 and 81201212) and the Education Department of Liaoning Province (grant no. L2014360). Professor Han Liu is funded by the "Climbing Scholar" and "Excellent Talents" schemes of Liaoning Province, China.

\section{References}

1. Wang Q, Huang C, Xue $M$ and Zhang X: Expression of endogenous BMP-2 in periosteal progenitor cells is essential for bone healing. Bone 48: 524-532, 2011.

2. Wilusz RE, Sanchez-Adams J and Guilak F: The structure and function of the pericellular matrix of articular cartilage. Matrix Biol 39: 25-32, 2014.

3. Demoor M, Ollitrault D, Gomez-Leduc T, Bouyoucef M, Hervieu M, Fabre H, Lafont J, Denoix JM, Audigié F, Mallein-Gerin F, et al: Cartilage tissue engineering: Molecular control of chondrocyte differentiation for proper cartilage matrix reconstruction. Biochim Biophys Acta 1840: 2414-2440, 2014.

4. Jiang $\mathrm{Y}$ and Tuan RS: Origin and function of cartilage stem/progenitor cells in osteoarthritis. Nat Rev Rheumatol 11: 206-212, 2015

5. Rodrigues MT, Gomes ME and Reis RL: Current strategies for osteochondral regeneration: From stem cells to pre-clinical approaches. Curr Opin Biotechnol 22: 726-733, 2011.

6. Kock L, van Donkelaar CC and Ito K: Tissue engineering of functional articular cartilage: The current status. Cell Tissue Res 347: 613-627, 2012.

7. van Osch GJ, Brittberg M, Dennis JE,Bastiaansen-Jenniskens YM, Erben RG, Konttinen YT and Luyten FP: Cartilage repair: Past and future--lessons for regenerative medicine. J Cell Mol Med 13: 792-810, 2009.

8. Edwards PK, Ackland T and Ebert JR: Clinical rehabilitation guidelines for matrix-induced autologous chondrocyte implantation on the tibiofemoral joint. J Orthop Sports Phys Ther 44: 102-119, 2014.

9. Yu SM and Kim SJ: The thymoquinone-induced production of reactive oxygen species promotes dedifferentiation through the ERK pathway and inflammation through the p38 and PI3K pathways in rabbit articular chondrocytes. Int J Mol Med 35: 325-332, 2015.

10. Goessler UR, Hörmann K and Riedel F: Tissue engineering with chondrocytes and function of the extracellular matrix (Review) Int J Mol Med 13: 505-513, 2004.

11. Foldager CB: Advances in autologous chondrocyte implantation and related techniques for cartilage repair. Dan Med J 60: B4600, 2013.

12. Yanagawa T, Funasaka T, Tsutsumi S, Watanabe H and Raz A: Novel roles of the autocrine motility factor/phosphoglucose isomerase in tumor malignancy. Endocr Relat Cancer 11: 749-759, 2004

13. Funasaka $\mathrm{T}$ and $\operatorname{Raz} \mathrm{A}$ : The role of autocrine motility factor in tumor and tumor microenvironment. Cancer Metastasis Rev 26: $725-735,2007$
14. Tsutsumi S, Hogan V, Nabi IR and Raz A: Overexpression of the autocrine motility factor/phosphoglucose isomerase induces transformation and survival of NIH-3T3 fibroblasts. Cancer Res 63: 242-249, 2003.

15. Shih WL, Liao MH, Lin PY, Chang CI, Cheng HL, Yu FL and Lee JW: PI 3-kinase/Akt and STAT3 are required for the prevention of TGF-beta-induced Hep3B cell apoptosis by autocrine motility factor/phosphoglucose isomerase. Cancer Lett 290: 223-237, 2010.

16. Kho DH, Nangia-Makker P, Balan V, Hogan V, Tait L, Wang Y and Raz A: Autocrine motility factor promotes HER2 cleavage and signaling in breast cancer cells. Cancer Res 73: 1411-1419, 2013.

17. Tsutsumi S, Yanagawa T, Shimura T, Fukumori T, Hogan V, Kuwano $\mathrm{H}$ and Raz A: Regulation of cell proliferation by autocrine motility factor/phosphoglucose isomerase signaling. J Biol Chem 278: 32165-32172, 2003.

18. Silletti S and Raz A: Autocrine motility factor is a growth factor. Biochem Biophys Res Commun 194: 446-457, 1993.

19. Zhi J, Sommerfeldt DW, Rubin CT and Hadjiargyrou M: Differential expression of neuroleukin in osseous tissues and its involvement in mineralization during osteoblast differentiation. J Bone Miner Res 16: 1994-2004, 2001.

20. Li Y, Qin J, Lin B and Zhang W: The effects of insulin-like growth factor-1 and basic fibroblast growth factor on the proliferation of chondrocytes embedded in the collagen gel using an integrated microfluidic device. Tissue Eng Part C Methods 16: $1267-1275,2010$

21. Spahn G, Kahl E, Muckley T, Hofmann GO and Klinger HM: Arthroscopic knee chondroplasty using a bipolar radiofrequency-based device compared to mechanical shaver: Results of a prospective, randomized, controlled study. Knee Surg Sports Traumatol Arthrosc 16: 565-573, 2008.

22. Gelse K, von der Mark K, Aigner T, Park J and Schneider H: Articular cartilage repair by gene therapy using growth factor-producing mesenchymal cells. Arthritis Rheum 48: 430-441, 2003.

23. Haene R, Qamirani E, Story RA, Pinsker E and Daniels TR: Intermediate outcomes of fresh talar osteochondral allografts for treatment of large osteochondral lesions of the talus. J Bone Joint Surg Am 94: 1105-1110, 2012.

24. Nukavarapu SP and Dorcemus DL: Osteochondral tissue engineering: Current strategies and challenges. Biotechnol Adv 31: 706-721, 2013.

25. Chan BP and Leong KW: Scaffolding in tissue engineering: General approaches and tissue-specific considerations. Eur Spine J 17 Suppl 4: 467-479, 2008.

26. Cheng CW, Solorio LD and Alsberg E: Decellularized tissue and cell-derived extracellular matrices as scaffolds for orthopaedic tissue engineering. Biotechnol Adv 32: 462-484, 2014.

27. Krejci P, Masri B, Fontaine V, Mekikian PB, Weis M, Prats H and Wilcox WR: Interaction of fibroblast growth factor and $\mathrm{C}$-natriuretic peptide signaling in regulation of chondrocyte proliferation and extracellular matrix homeostasis. J Cell Sci 118: 5089-5100, 2005

28. Phornphutkul C, Wu KY, Yang X, Chen Q and Gruppuso PA: Insulin-like growth factor-I signaling is modified during chondrocyte differentiation. J Endocrinol 183: 477-486, 2004.

29. Bobick BE and Kulyk WM: The MEK-ERK signaling pathway is a negative regulator of cartilage-specific gene expression in embryonic limb mesenchyme. J Biol Chem 279: 4588-4595, 2004.

30. Gikas PD, Bayliss L, Bentley G and Briggs TW: An overview of autologous chondrocyte implantation. J Bone Joint Surg Br 91: 997-1006, 2009.

31. Araki K, Shimura T, Yajima T, Tsutsumi S, Suzuki H, Okada K, Kobayashi T, Raz A and Kuwano H: Phosphoglucose isomerase/autocrine motility factor promotes melanoma cell migration through ERK activation dependent on autocrine production of interleukin-8. J Biol Chem 284: 32305-32311, 2009.

32. Niizeki H, Kobayashi M, Horiuchi I, Akakura N, Chen J, Wang J, Hamada JI, Seth P, Katoh H, Watanabe H, et al: Hypoxia enhances the expression of autocrine motility factor and the motility of human pancreatic cancer cells. Br J Cancer 86: 1914-1919, 2002.

33. Timar J, Trikha M, Szekeres K, Bazaz R, Tovari J, Silletti S, Raz A and Honn KV: Autocrine motility factor signals integrin-mediated metastatic melanoma cell adhesion and invasion. Cancer Res 56: 1902-1908, 1996. 
34. Niinaka Y, Paku S, Haga A, Watanabe H and Raz A: Expression and secretion of neuroleukin/phosphohexose isomerase/maturation factor as autocrine motility factor by tumor cells. Cancer Res 58: 2667-2674, 1998.

35. Dobashi Y, Watanabe H, Matsubara M, Yanagawa T, Raz A, Shimamiya $T$ and Ooi A: Autocrine motility factor/glucose-6-phosphate isomerase is a possible predictor of metastasis in bone and soft tissue tumours. J Pathol 208: 44-53, 2006.

36. Luo Y, Long JM, Lu C, Chan SL, Spangler EL, Mascarucci P, Raz A, Longo DL, Mattson MP, Ingram DK and Weng NP: A link between maze learning and hippocampal expression of neuroleukin and its receptor gp78. J Neurochem 80: 354-361, 2002.

37. Yang Y, Cheng XR, Zhang GR, Zhou WX and Zhang YX: Autocrine motility factor receptor is involved in the process of learning and memory in the central nervous system. Behav Brain Res 229: 412-418, 2012.

38. Funasaka T, Haga A, Raz A and Nagase H: Tumor autocrine motility factor is an angiogenic factor that stimulates endothelial cell motility. Biochem Biophys Res Commun 285: 118-128, 2001.

39. Bhise NS, Ribas J, Manoharan V, Zhang YS, Polini A, Massa S, Dokmeci MR and Khademhosseini A: Organ-on-a-chip platforms for studying drug delivery systems. J Control Release 190: 82-93, 2014.
40. Björnmalm M, Yan Y and Caruso F: Engineering and evaluating drug delivery particles in microfluidic devices. J Control Release 190: 139-149, 2014

41. Ye N, Qin J, Shi W, Liu X and Lin B: Cell-based high content screening using an integrated microfluidic device. Lab Chip 7: 1696-1704, 2007.

42. Kane BJ, Zinner MJ, Yarmush ML and Toner M: Liver-specific functional studies in a microfluidic array of primary mammalian hepatocytes. Anal Chem 78: 4291-4298, 2006.

43. Tourovskaia A, Figueroa-Masot X and Folch A: Differentiation-on-a-chip: A microfluidic platform for long-term cell culture studies. Lab Chip 5: 14-19, 2005.

44. Zhong W, Tian K, Zheng X, Li L, Zhang W, Wang S and Qin J: Mesenchymal stem cell and chondrocyte fates in a multishear microdevice are regulated by Yes-associated protein. Stem Cells Dev 22: 2083-2093, 2013.

45. Sanz-Ramos P, Duart J, Rodríguez-Goñi MV, Vicente-Pascual M, Dotor J, Mora G and Izal-Azcárate I: Improved Chondrogenic Capacity of Collagen Hydrogel-Expanded Chondrocytes: In Vitro and in Vivo Analyses. J Bone Joint Surg Am 96: 1109-1117, 2014.

46. Bobick BE, Chen FH, Le AM and Tuan RS: Regulation of the chondrogenic phenotype in culture. Birth Defects Res C Embryo Today 87: 351-371, 2009. 